\title{
THE TRIVIAL HOMOTOPY CLASS OF MAPS FROM TWO-COMPLEXES INTO THE REAL PROJECTIVE PLANE
}

\author{
Marcio Colombo Fenille
}

\begin{abstract}
We study reasons related to two-dimensional CW-complexes which prevent an extension of the Hopf-Whitney Classification Theorem for maps from those complexes into the real projective plane, even in the simpler situation in which the complex has trivial second integer cohomology group. We conclude that for such a two-complex $K$, the following assertions are equivalent: (1) Every based map from $K$ into the real projective plane is based homotopic to a constant map; (2) The skeleton pair $\left(K, K^{1}\right)$ is homotopy equivalent to that of a model two-complex induced by a balanced group presentation; (3) The number of two-dimensional cells of $K$ is equal to the first Betti number of its one-skeleton; (4) $K$ is acyclic; (5) Every based map from $K$ into the circle $S^{1}$ is based homotopic to a constant map.
\end{abstract}

\section{Introduction and main theorem}

Given topological spaces $X$ and $Y$, we denote the set of homotopy classes of maps from $X$ into $Y$ by $[X ; Y]$. If $X$ and $Y$ have base points $x_{*}$ and $y_{*}$, respectively, let $[X ; Y]_{*}$ denote the based homotopy classes of based maps from $X$ into $Y$, that is, maps $\left(X, x_{*}\right) \rightarrow\left(Y, y_{*}\right)$. If the base point $x_{*} \in X$ is nondegenerated (in particular, if $X$ is a $\mathrm{CW}$-complex) and $Y$ is path connected, then there exists a natural action of the fundamental group $\pi_{1}\left(Y, y_{*}\right)$ on the set

2010 Mathematics Subject Classification. 55Q05, 55Q55.

Key words and phrases. Hopf-Whitney Classification Theorem, two-dimensional CW complexes, group presentation, homotopy class, cohomology group, real projective plane.

This work is sponsored by FAPEMIG - Grant CEX-APQ-00130-11. 
$[X ; Y]_{*}$ in such a way that $[X ; Y]$ is the quotient set of $[X ; Y]_{*}$ by this action. See [4, Theorem 6.57]. As a particular consequence, a based map is null-homotopic if and only if it is based null-homotopic. See [4, Corollary 6.58]. Furthermore, if $Y$ is simply connected, there exists a bijection between $[X ; Y]$ and $[X ; Y]_{*}$. See $[4, \S 6.16]$ for details.

The Hopf-Whitney Classification Theorem [8, Corollary 6.19, p. 244] states that the set $[K ; Y]$ of the homotopy classes of maps from an $n$-dimensional CW-complex $K$ into an $(n-1)$-connected $n$-simple space $Y$ is in one-to-one correspondence with the cohomology group $H^{n}\left(K ; \pi_{n}(Y)\right)$. We remark that for $n \geq 2$, the hypothesis of $Y$ to be $(n-1)$-connected implies automatically that $Y$ is also $n$-simple, since in this case $Y$ is simply connected. Again under such conditions, we have a one-to-one correspondence between $[K ; Y]$ and the set $[K ; Y]_{*}$ of the based homotopy classes, as we noted at the end of the first paragraph. Therefore, for $n \geq 2$, we might replace $[K ; Y]$ by $[K ; Y]_{*}$ in the statement of the Hopf-Whitney Classification Theorem.

Obviously, the Hopf-Whitney Classification Theorem does not apply for a two-dimensional CW-complex $K$ (shortly a two-complex $K$ ) and $Y=\mathbb{R P}^{2}$, since the real projective plane $\mathbb{R P}^{2}$ is not 1-connected (simply connected). In fact, we may provide easily examples in which the cohomology group

$$
H^{2}\left(K ; \pi_{2}\left(\mathbb{R P}^{2}\right)\right)=H^{2}(K ; \mathbb{Z})=H^{2}(K)
$$

is "smaller" than the set $\left[K ; \mathbb{R P}^{2}\right]_{*}$ of based homotopy classes of maps from $K$ into $\mathbb{R P}^{2}$. This happens, for instance, if $K$ collapses to a bouquet $\bigvee^{n} S^{1}$ of $n \geq 1$ circles; in this case, $H^{2}(K)=0$, although the set $\left[K ; \mathbb{R P}^{2}\right]_{*}$ has $2^{n}$ elements. For another example, one in which the two-complex $K$ does not collapses to a one-complex, consider $K=S^{1} \vee S^{2}$; in this case we have $H^{2}(K) \approx \mathbb{Z}$ whereas $\left[K ; \mathbb{R P}^{2}\right]_{*}$ contains isomorphic copies of $\mathbb{Z}$ and $\mathbb{Z}_{2}$. The fact $H^{2}(K)$ is "smaller" than $\left[K ; \mathbb{R P}^{2}\right]_{*}$ is general; in fact, Lemma 3.1 shows that for a two-complex $K$ with fundamental group $\Pi$, there exists a one-to-one correspondence between $H^{2}(K)$ and $\left[K ; \mathbb{R P}^{2}\right]_{*}$ if and only if $\operatorname{Hom}\left(\Pi ; \mathbb{Z}_{2}\right)=0$; else, there exists a strictly injection from $H^{2}(K)$ into $\left[K ; \mathbb{R P}^{2}\right]_{*}$. In any case, we have

$$
\left[K ; \mathbb{R P}^{2}\right]_{*}=0 \Rightarrow H^{2}(K)=0,
$$

where the expression on the left side means that $\left[K ; \mathbb{R P}^{2}\right]_{*}$ contains only the based homotopy class of the constant map at the base point of $\mathbb{R P}^{2}$.

In this article we study conditions on a finite and connected two-complex $K$ to ensure the opposite implication, that is, we study the possible triviality of the set $\left[K ; \mathbb{R P}^{2}\right]_{*}$ when $K$ is a finite and connected two-dimensional CW-complex with trivial second cohomology group. For such a two-complex, we do not have always $\left[K ; \mathbb{R P}^{2}\right]_{*}=0$, but we may characterize the two-complexes for which this happens. Such characterization is our main theorem: 